\title{
Minireview
}

\section{Comparing cellular proteomes by mass spectrometry}

\author{
Florian Fröhlich and Tobias C Walther
}

\begin{abstract}
Address: Organelle Architecture and Dynamics, Max Planck Institute of Biochemistry, Am Klopferspitz 18, 82152 Martinsried/Munich, Germany.

Correspondence: Tobias C Walther. Email: twalther@biochem.mpg.de
\end{abstract}

\begin{abstract}
Mass spectrometry and cryo-electron tomography together enable the determination of the absolute and relative abundances of proteins and their localization, laying the groundwork for comprehensive systems analyses of cells.
\end{abstract}

Biological systems are characterized by the dynamic interplay of their components, and to understand how individual parts act together it is crucial to know the composition of a system and how it changes over time. The protein components are of prime interest as they provide structure and carry out many functions in the cell. The transcriptome has been much used as a proxy to infer changes in protein expression, as techniques for measuring global RNA levels preceded those for measuring the proteome. However, when the levels of an mRNA and its corresponding protein are systematically compared, many differences in their abundance emerge, resulting in poor quantitative correlation overall between transcriptome and proteome [1-3]. Ways of measuring protein levels directly are therefore highly desirable, and breakthroughs in mass spectrometry (MS)based proteomics are starting to enable this on a global scale.

In experiments recently published in Nature, Ruedi Aebersold and colleagues (Malmström et al. [4]) combined MS-based measurements of protein abundance in the bacterial pathogen Leptospira interrogans, the agent of Weil's disease, with imaging by cryo-electron tomography (CET) of distinct structures of known protein composition, such as the flagellar motor (in which the precise number and type of the protein subunits can be counted). The CET imaging provided a way of confirming the MS proteinquantitation data. The protein-abundance measurements then enabled the effect of the antibiotic ciprofloxacin on a large fraction of the Leptospira proteome to be determined. In this article we describe some of the recent developments in MS-based proteomics that enable such experiments, focusing on quantitative techniques that will eventually allow a complete inventory of cellular proteins. The goal for proteomics is the measurement of the absolute and relative abundances of proteins at high accuracy and with minimal effort. But currently this means a compromise between depth of analysis and measurement time.

\section{Identifying proteins by mass spectrometry}

Intact proteins are difficult to identify by MS because their sequence cannot be obtained by fragmentation and so MS-based proteomics relies on analysis of peptides obtained by proteinase digestion of the sample. By analogy with genome-sequencing methods, this approach has been called 'shotgun' proteomics. The resulting peptide mixtures are dauntingly complex and are fractionated before submitting them to MS. Several recent studies, including the determination of the yeast and Leptospira proteomes $[2,4]$, used isoelectric focusing in so-called OFF-gels $[5,6]$ as a first separation step. Following this initial fractionation, peptides are separated by liquid chromatography (LC) most commonly directly coupled to electrospray ionization of peptides (ESI) or less frequently to matrixassisted laser desorption ionization (MALDI) to produce ions for MS.

In the next step, mass-to-charge $(\mathrm{m} / \mathrm{z})$ values of peptides and their ion intensities are determined by $\mathrm{MS}\left(\mathrm{MS}^{1}\right.$ or 'parent ion' spectra). To reliably identify peptides, the (typically) 5 to 20 most abundant peptides are selected for further fragmentation, resulting in a sequence-characteristic spectrum ( $\mathrm{MS}^{2}$ or fragmentation spectrum) for each peptide that is used to search databases to identify the peptide (Figure 1a). In the determination of the Leptospira proteome, Malmström et al. [4] collected more than 415, $000 \mathrm{MS}^{2}$ spectra that could be assigned to more than 18 ,ooo unique peptides, leading to the identification of 2,221 proteins ( $61 \%$ of the predicted open reading frames). To analyze the complex peptide mixtures typical of proteomics very high mass resolution is required. Otherwise, MS spectra from different peptides overlap, making peptide identification and quantification potentially inaccurate and unreliable. Precision instruments, in particular orbital frequency resonance ion traps such as the Orbitrap [7], are therefore most widely used for proteomics.

\section{Methods for comparative quantitative proteomics}

A common goal in proteomics is the accurate quantification and comparison of the proteomes of cells in different physiological or developmental states. For Leptospira, the 
(a)

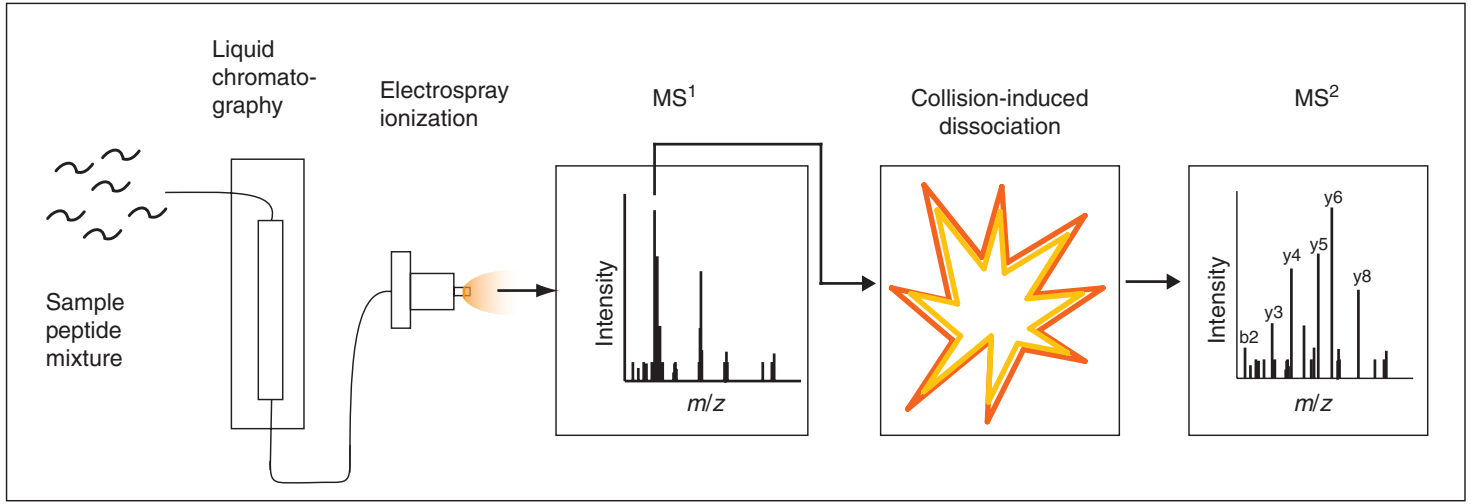

(b)

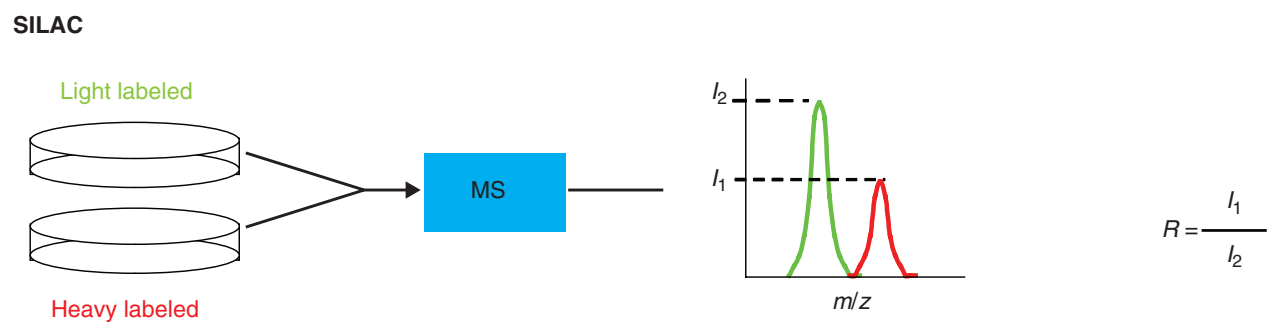

'Label-free’ quantitation
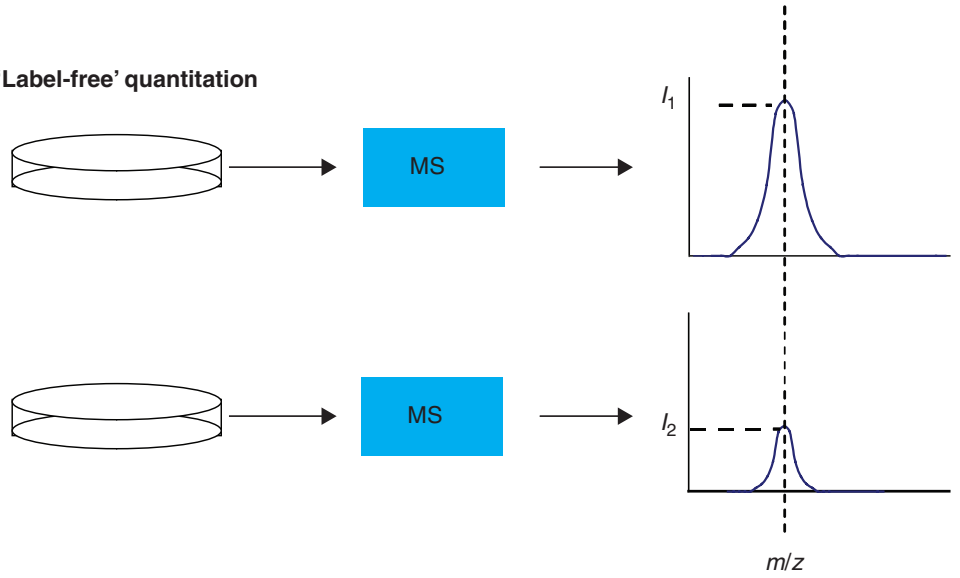

$R=\frac{l_{1}}{I_{2}}$

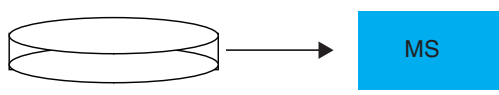

Absolute quantitation with standard peptides

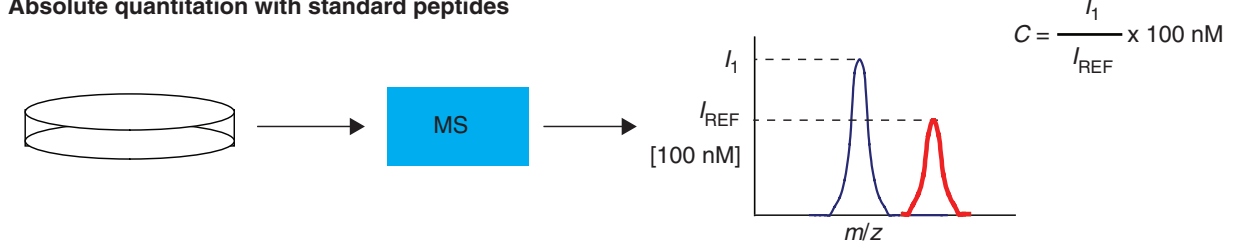

Figure 1 
Figure 1 continued

Quantitative MS-based proteomics. (a) Analysis of complex peptide mixtures by LC-MS². Peptide mixtures are resolved by liquid chromatography, ionized through electrospray and resolved by MS ${ }^{1}$. Selected peptides are fragmented by collision with an inert gas and the resulting $\mathrm{MS}^{2}$ spectra are recorded. (b) Quantitative proteomics strategies. In the SILAC technique, isotope-labeled peptide intensities (I) are compared in the $\mathrm{MS}^{1}$ spectra. For 'label-free' quantitation, intensities of peptides are compared between different runs. Alternatively, standard peptides are spiked into the mixture to yield calibration for absolute peptide abundances. $R$ refers to the ratio between either heavy and light peptides (SILAC panel) or ion intensities between different runs (label-free quantitation).

interesting question addressed by Malmström et al. [4] is how the proteome reacts to addition of an antibiotic. They took the approach of quantifying protein abundance directly using a label-free method, which we shall discuss later. Another approach would have been to derivatize the peptides from different conditions with isobaric labels that yield different, indicative, small molecules after fragmentation, a technique called isobaric tag for relative and absolute quantitation (iTRAQ) [8]. After fragmentation these derivatives yield distinctive small molecules indicative of the peptide. In such an experiment, the relative abundance of these indicators is used to quantify the relative abundance of the different peptides (and thus proteins) in the sample.

Metabolic labeling of proteins yields similar information, but avoids complications of in vitro coupling such as incomplete reactions. Samples are labeled in vivo with amino acids (lysine and arginine) labeled with heavy nonradioactive isotopes such as ${ }^{13} \mathrm{C}$ or ${ }^{15} \mathrm{~N}$, and compared with samples containing unlabeled amino acids, a technique called stable isotope labeling of cells in culture (SILAC) [9]. Peptides are then generated by digesting with proteinases (for example, trypsin) that cut specifically after labeled amino acids, thereby ensuring that each peptide contains at least one labeled amino acid. This results in a distinct shift in MS spectra between heavy and light peptides. The intensity ratio between peaks in a SILAC pair indicates the abundance ratio of proteins from which the peptides were derived (Figure $1 b$ ).

For more accurate measurements, multiple peptides from a protein are typically averaged and this analysis is now completely automated [10]. Because of the high resolving power of Orbitrap mass spectrometers, this methodology can be applied to very complex mixtures and closely spaced peaks can be well resolved. Together with only one previous fractionation step - isoelectric focusing - this experimental setup was used for the first quantitation of a eukaryotic proteome, that of Saccharomyces cerevisiae, in the haploid and diploid phases of the life cycle $(4,399$ proteins were identified and 4,033 quantitated from $1,788,451$ SILAC pair peptides [2]). If the abundances of at least some proteins are known, as was the case in yeast, they can be used to calibrate the MS data and yield absolute protein measurements. Advantages of this approach include very accurate quantitation and the fact that no previous knowledge of proteins that change in abundance is required. This is in contrast to the classical proteindetection methods, for example, immunoblotting, where reagents are often limiting and a clear hypothesis about which protein(s) to measure is required. SILAC, pioneered by the Mann laboratory, is now widely used for protein analyses in yeast, flies and even mice $[1,2,11,12]$.

\section{Label-free approaches}

A limitation of SILAC experiments is that labeling is necessary but is not always possible - for example in human samples. One option is to compare SILAC-labeled reference extracts or recombinant proteins against samples of interest [13]. Alternatively, it may be desirable to find means of reliably quantifying protein abundance directly, an approach taken by Malmström et al. [4] for the characterization of Leptospira and its reaction to ciprofloxacin. Early methods of 'label-free' quantification used the frequency of peptide selection for fragmentation as a measure of their abundance - termed 'spectral counting' $[14,15]$. Because that technique uses an indirect measurement for peptide abundance and only works reliably for proteins with many available peptides, alternatives have been developed. Specifically, peptide-ion intensities in the parent $\mathrm{MS}^{1}$ spectrum are used to quantify peptide abundances. For this method, reproducible identification of the same peptides in different LC-MS runs is crucial (Figure 1b). This is achieved by high massaccuracy measurements, and also by aligning different runs based on the LC retention time of matched peptides between them [16]. Although still somewhat less accurate than quantification methods relying on isotope labels, this methodology makes a variety of clinical and environmental samples accessible, such as cancer or other biopsies.

In a series of papers including the Leptospira study, the peptide-ion intensity method has been further developed to calibrate MS measurements and yield absolute quantifications $[4,6,17,18]$. As standards for calibration, isotopelabeled reference peptides are spiked into samples. Comparison of the ion intensities of standards of known abundance and of the experimental peptides yields an absolute concentration for the latter (Figure $1 \mathrm{~b}$ ). In very complex mixtures, it can be difficult to detect such peptide pairs, but in principle, advances in instrumentation and development of analytic tools should eventually allow the measurement of most peptides in a mixture, including those spiked as a reference. In the meantime, targeted approaches such as selected reaction monitoring (SRM) 
are promising. In these experiments, a series of mass analyzers (for example, a triple quadrupole MS) 'filters' only targeted peptides. In combination with isotopelabeled standards, the abundance of peptides is quantitated by comparison of parent ion pair intensities. As a result of effective filtering, SRM assays are performed very fast and can monitor a series of peptides. To obtain a calibration curve for the Leptospira proteome that can be extrapolated to determine the absolute abundances of all detected proteins, Malmström et al. [4] used 19 peptides to report on proteins ranging in abundance from 40 to 15,000 copies per cell. One appeal of this methodology is the rapid monitoring of a limited number of proteins, which would enable a comparison of abundance in many samples and the characterization of protein dynamics over time.

A potential problem with the peptide-ion intensity method is that parent ion scans are usually carried out using quadrupoles with high sensitivity and dynamic range but low mass accuracy, possibly leading to overlapping peaks and convolution of signals when analyzing complex mixtures. A remedy for this could be to acquire full highresolution spectra by scanning MS and then select peptides for sequencing by an 'inclusion' list. Satisfyingly, in the case of Leptospira [4], the quantitation obtained using an SRM-derived calibration curve agreed very well with the counting by CET of the subunits in prominent cellular structures such as the flagella and the flagellar motor, or of methyl-accepting proteins in individual cells. This work shows how MS-based proteomics combined with highresolution CET can yield information on protein abundance and localization.

Having obtained accurate measurements of the levels of individual proteins, it is then possible to compare proteomes under different physiological conditions. In the case of Leptospira [4], the comparison showed that the bacterium reacts to ciprofloxacin by strongly inducing the expression of a number of proteins (whose existence was previously only predicted from the genome sequence), but maintains overall protein concentration. The upregulated proteins might include interesting targets for combination therapy and the experiment shows in principle how this technology can be used for an unbiased systems characterization.

Over the past decade, developments in MS-based proteomics have greatly accelerated. In particular, new instrumentation and automation of MS-spectra interpretation enables the quantification of essentially whole-organism proteomes in single experiments. Tools to calibrate measurements are already leading to the determination of absolute protein abundances and specialized methods can be used to target subsets of proteins. All together, these developments predict that MS-based proteomics will become a staple technique in systems biology.

\section{Acknowledgements}

We thank Bob Farese, Natalie Krahmer and members of the Walther lab for discussions and contributions to this essay. This work was supported by the Max Planck Society, the German Research Council (DFG) and the Human Frontier Science Program (HFSP).

\section{References}

1. Bonaldi T, Straub T, Cox J, Kumar C, Becker PB, Mann M: Combined use of RNAi and quantitative proteomics to study gene function in Drosophila. Mol Cell 2008, 31:762772.

2. de Godoy LM, Olsen JV, Cox J, Nielsen ML, Hubner NC, Frohlich F, Walther TC, M Mann M: Comprehensive massspectrometry-based proteome quantification of haploid versus diploid yeast. Nature 2008, 455:1251-1254.

3. Ideker T, Thorsson V, Ranish JA, Christmas R, Buhler J, Eng JK, Bumgarner R, Goodlett DR, Aebersold R, Hood L: Integrated genomic and proteomic analyses of a systematically perturbed metabolic network. Science 2001, 292:929934.

4. Malmström J, Beck M, Schmidt A, Lange V, Deutsch EW, Aebersold R: Proteome-wide cellular protein concentrations of the human pathogen Leptospira interrogans. Nature 2009, 460:762-765.

5. Hubner NC, Ren S, Mann M: Peptide separation with immobilized pl strips is an attractive alternative to in-gel protein digestion for proteome analysis. Proteomics 2008, 8:48624872.

6. Picotti P, Bodenmiller B, Mueller LN, Domon B, Aebersold R: Full dynamic range proteome analysis of $S$. cerevisiae by targeted proteomics. Cell 2009, 138:795-806.

7. Hu Q, Noll RJ, Li H, Makarov A, Hardman M, Graham Cooks R: The Orbitrap: a new mass spectrometer. J Mass Spectrom 2005, 40:430-443.

8. Ross PL, Huang YN, Marchese JN, Williamson B, Parker K, Hattan S, Khainovski N, Pillai S, Dey S, Daniels S, Purkayastha $S$, Juhasz $P$, Martin S, Bartlet-Jones M, He F, Jacobson A, Pappin DJ: Multiplexed protein quantitation in Saccharomyces cerevisiae using amine-reactive isobaric tagging reagents. Mol Cell Proteomics 2004, 3:1154-1169.

9. Ong SE, Blagoev B, Kratchmarova I, Kristensen DB, Steen H, Pandey A, Mann M: Stable isotope labeling by amino acids in cell culture, SILAC, as a simple and accurate approach to expression proteomics. Mol Cell Proteomics 2002, 1:376386.

10. Cox J, Mann M: MaxQuant enables high peptide identification rates, individualized p.p.b.-range mass accuracies and proteome-wide protein quantification. Nat Biotechnol 2008, 26:1367-1372.

11. Kruger M, Moser M, Ussar S, Thievessen I, Luber CA, Forner F, Schmidt S, Zanivan S, Fassler R, Mann M: SILAC mouse for quantitative proteomics uncovers kindlin-3 as an essential factor for red blood cell function. Cell 2008, 134: 353-364.

12. Liao L, Park SK, $\mathrm{Xu} T$, Vanderklish $P$, Yates JR 3rd: Quantitative proteomic analysis of primary neurons reveals diverse changes in synaptic protein content in fmr1 knockout mice. Proc Natl Acad Sci USA 2008, 105: 15281-15286.

13. Hanke S, Besir H, Oesterhelt D, Mann M: Absolute SILAC for accurate quantitation of proteins in complex mixtures down to the attomole level. J Proteome Res 2008, 7:11181130.

14. Liu H, Sadygov RG, Yates JR 3rd: A model for random sampling and estimation of relative protein abundance in shotgun proteomics. Anal Chem 2004, 76:4193-4201.

15. MacCoss MJ, Wu CC, Liu H, Sadygov R, Yates JR 3rd: A correlation algorithm for the automated quantitative analysis of shotgun proteomics data. Anal Chem 2003, 75:69126921.

16. Strittmatter EF, Ferguson PL, Tang K, Smith RD: Proteome analyses using accurate mass and elution time peptide 
tags with capillary LC time-of-flight mass spectrometry. $J$ Am Soc Mass Spectrom 2003, 14:980-991.

17. Gerber SA, Rush J., Stemman O, Kirschner MW, Gygi SP: Absolute quantification of proteins and phosphoproteins from cell lysates by tandem MS. Proc Natl Acad Sci USA 2003, 100:6940-6945.

18. Silva JC, Gorenstein MV, Li GZ, Vissers JP, Geromanos SJ: Absolute quantification of proteins by LCMSE: a virtue of parallel MS acquisition. Mol Cell Proteomics 2006, 5:144156.

Published: 28 October 2009

doi:10.1186/gb-2009-10-10-240

(c) 2009 BioMed Central Ltd 